\title{
A new parallel high-pressure packing system enables rapid multiplexed production of capillary columns
}

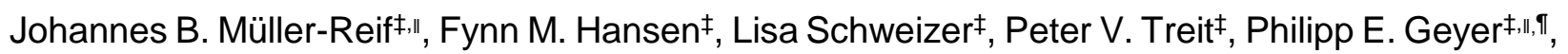
Matthias Mann ${ }^{\ddagger}$, ,**

\section{Affiliations}

‡Department of Proteomics and Signal Transduction, Max Planck Institute of Biochemistry, Martinsried, Germany INNF Center for Protein Research, Faculty of Health Sciences, University of Copenhagen, Copenhagen, Denmark "New address: OmicEra Diagnostics GmbH, Planegg, Germany

${ }^{*}$ Corresponding author. Tel: +49 898578 2557; E-mail: mmann@biochem.mpg.de

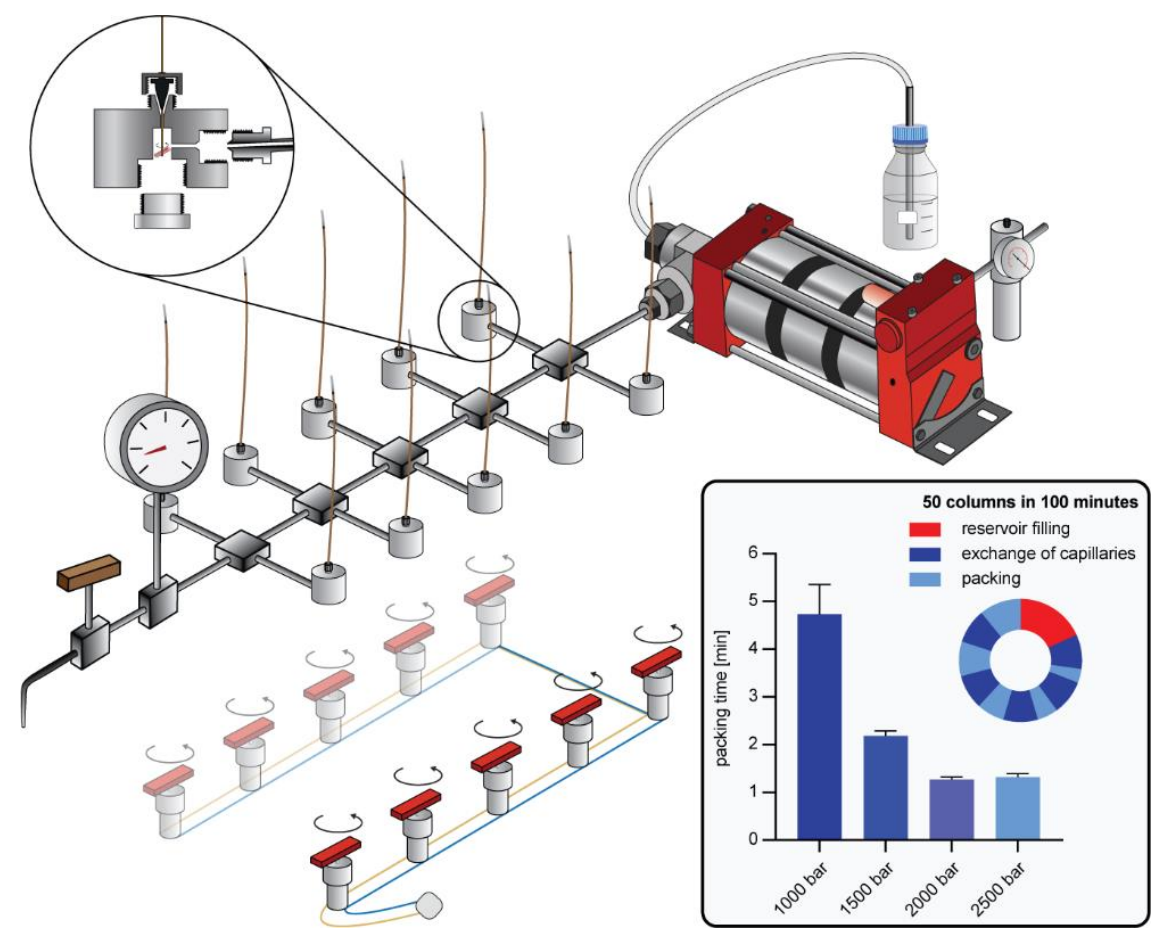




\section{Abstract}

Reversed-phase high performance liquid chromatography (HPLC) is the most commonly applied peptide separation technique in mass spectrometry (MS)-based proteomics. Particle-packed capillary columns are predominantly used in nano-flow HPLC systems. Despite being the broadly applied standard for many years capillary columns are still expensive and suffer from short lifetimes, particularly in combination with ultra-high-pressure chromatography systems. For this reason, and to achieve maximum performance, many laboratories produce their own inhouse packed columns. This typically requires a considerable amount of time and trained personnel. Here, we present a new packing system for capillary columns enabling rapid, multiplexed column production with pressures reaching up to 3000 bar. Requiring only a conventional gas pressure supply and methanol as driving fluid, our system replaces the traditional setup of helium pressured packing bombs. By using 10x multiplexing, we have reduced the production time to just under 2 minutes for several 50 cm columns with $1.9 \mu \mathrm{m}$ particle size, speeding up the process of column production 40 to 800 times. We compare capillary columns with various inner diameters (ID) and length packed under different pressure conditions with our newly designed, broadly accessible high-pressure packing station.

One sentence summary: A newly constructed parallel high-pressure packing system enables the rapid multiplexed production of capillary columns.

\section{Introduction}

State-of-the-art mass spectrometry (MS)-based proteomic pipelines typically consist of a sample preparation workflow to digest proteins and harvest pure peptides, a liquid chromatography (LC) system for peptide separation, a mass spectrometer and a sophisticated bioinformatics pipeline for raw data interpretation and subsequent statistical analysis $(1,2)$. The LC system plays a central role by separating the complex mixture of tens of thousands of peptides in a time-resolved manner according to their biochemical properties, making them ultimately manageable for the MS system over the course of a gradient $(3,4)$. The most widely applied technique for highperformance applications is reversed-phase separation, originally introduced in the 1970s (5). In essence, chromatographic systems are made of programmable pumps with the ability to form a gradient of a mixture of different agents. In the case of reversed-phase LC, the stationary phase is nonpolar, separating analytes by hydrophobicity over the course of a gradient of increasing nonpolar mobile phase. The LC system is coupled to the mass spectrometer by electrospray (ES) ionization via an emitter (6). Glass or steel needles are commonly connected to the column. Particle packed capillaries for chromatography can also be used for ES without being coupled to an additional emitter (7-9). These basic attributes are shared by most LC-MS systems and differences are mainly defined by operational flow. Nanoflow LC operates at flow rates of several hundred nanoliters per minute and is the standard in proteomics due to the high sensitivity obtainable.

High flow rates in the $\mu \mathrm{l}$ to $\mathrm{ml}$ range, applied to columns with large inner diameters, are typically used in high-throughput or industrial-scale analysis as well as analytical MS application areas. Although these micro-flow systems limit sensitivity, recent work has demonstrated robust and 
reproducible performance $(10,11)$. Reproducibility and stability of those systems are high, but drawbacks are lowered sensitivity and a need for high sample amounts. Compared to developments in sample preparation, MS instrumentation, scan modes and software, the LC apparatus has been largely unchanged in cutting edge MSbased proteomics. While identifications in proteomics experiments have doubled in single-shot experiments this can mainly be traced to improvement on the MS instrumentation and software (12-17). Current trends in LC developments aim rather towards systems for higher throughput and increasing robustness required for clinical applications (18), whereas the race for better separation in single-shot high performance runs with increasingly higher pump pressures has been comparatively abandoned. Consequently, a typically used setup for maximum sensitivity and performance for most experiments still consists of columns around $75 \mu \mathrm{m}$ ID with a length of 20 to $50 \mathrm{~cm}$, packed with sub $2 \mu \mathrm{m}$ particles. Although, better performance could be reached by longer columns or smaller particles, both conditions would result in higher operational pressures which tend to make the LC systems unstable $(4,19)$. For example, very high pressures can lead to leaks in the LC flow paths, resulting in poor reproducibility and subsequently a loss of measurement time.

Commercially available capillary columns in the aforementioned dimensions are expensive, especially considering how frequently they must be replaced. Therefore, many high-throughput laboratories produce packed capillaries in-house. Empty glass capillaries, ready to be packed and used, can either be purchased or produced from cheap polyimide coated capillaries using a laser puller. Typically, a gas pressure system is deployed to pack such columns with particles in the low $\mu \mathrm{m}$ range and instructions on the manufacturing process can be found online with open access (https://proteomicsresource.washington.edu/docs/protocol s05/Packing Capillary Columns.pdf). However, this process is inherently slow and interesting methods have recently been established with the aim of speeding up the packing process with high-pressure $(20)$ or dense beadslurry, as in the FlashPack method (21).

Combining these principles, we here present a highpressure packing system for capillary columns using a highconcentration bead slurry that has previously been described as beneficial for column performance (22). These high slurry concentrations and packing pressures of 1000 3000 bar allow us to achieve packing times for $50 \mathrm{~cm}$ columns in the minute range with our system, compared to hours for traditional procedures. Deploying a manifold system and a pump capable of high flow rates further multiplexes packing to up to 10 columns, making column production 40 to 800 times more time efficient compared to previous systems. We observe consistently good column performance for packing pressures at over 1000 bar with no adverse effects on the column backpressure and lifetime, while packing times continued to decrease at higher pressures. We provide a detailed blueprint of the system so it can readily be set up in interested laboratories (Suppl. Table 1).

\section{Experimental Procedures}

\section{Preparation of fused silica}

Fused silica from Polymicro® (TSP075365 for $75 \mu \mathrm{m}$ ID, TSP100365 for $100 \mu \mathrm{m}$ ID or TSP150365 for $150 \mu \mathrm{m}$ ID) was cut to $140 \mathrm{~cm}$. Polyimide coating was removed by a Bunsen burner and polishing with an ethanol-soaked tissue in the middle of the cut capillary at a width of $2 \mathrm{~cm}$. An electro spray emitter tip was pulled with a laser puller (Sutter P2000) at the polished part of the capillary resulting in two empty capillary columns ready to be packed. 


\section{Sample preparation: Protein digestion and in-StageTip purification}

HeLa cells were cultured in high glucose DMEM with $10 \%$ fetal bovine serum and $1 \%$ penicillin/streptomycin (all from Life Technologies, Inc.). Cells were counted using a countess cell counter (Invitrogen), and aliquots of $1 \times 10^{6}$ cells were washed twice with PBS (Life Technologies, Inc.), snap-frozen and stored at $-80^{\circ} \mathrm{C}$. Sample preparation was carried out with the PreOmics iST kit (www.preomics.de). We used one HeLa pellet with 1 million cells per cartridge, determinate the peptide concentration after peptide cleanup via NanoDrop and adjusted the peptide concentration to $0.2 \mathrm{mg} / \mathrm{ml}$.

\section{Ultra-high-pressure liquid chromatography and mass spectrometry}

Samples were measured using LC-MS instrumentation consisting of an EASY-nLC 1200 ultra-high-pressure system (Thermo Fisher Scientific), coupled to an Orbitrap Exploris 480 instrument (Thermo Fisher Scientific) using a nano-electrospray ion source (Thermo Fisher Scientific). Purified peptides were separated on high-pressure packed columns as described in the results section. For each LCMS/MS analysis with $75 \mu \mathrm{m}$ ID columns, $500 \mathrm{ng}$ peptides were used. For $100 \mu \mathrm{m}$ ID columns, $888 \mathrm{ng}$ peptides were used and for $150 \mu \mathrm{m}$ ID columns $2000 \mathrm{ng}$ peptides were used to adjust for the higher column volume.

Peptides were loaded in buffer $A^{*}(2 \%$ acetonitrile $(v / v)$, $0.1 \%$ trifluoroacetic acid $(\mathrm{v} / \mathrm{v}))$ and eluted with a linear 105 min gradient of $5-30 \%$ of buffer B $(0.1 \%$ formic acid, $80 \%$ (v/v) acetonitrile), followed by a $10 \mathrm{~min}$ increase to $95 \%$ of buffer B, followed by a 5 min wash of $95 \%$ buffer B. For the $75 \mu \mathrm{m}$ ID columns flow rate was $300 \mathrm{nl} / \mathrm{min}, 535 \mathrm{nl} / \mathrm{min}$ for $100 \mu \mathrm{m}$ ID columns and $1200 \mathrm{nI} / \mathrm{min}$ for $150 \mu \mathrm{m}$ ID columns to adjust for the linear flow velocity. Column temperature was kept at $60^{\circ} \mathrm{C}$ by an in-house-developed oven containing a Peltier element, and parameters were monitored in real time by the SprayQC software. MS data was acquired with a Top15 data-dependent MS/MS scan method. MS1 AGC Target was set to $300 \%$ in the $300-1650$ $\mathrm{m} / \mathrm{z}$ range with a maximum injection time of $25 \mathrm{~ms}$ and a resolution of 60,000 at $\mathrm{m} / \mathrm{z} 200$. Fragmentation of precursor ions was performed by higher-energy C-trap dissociation (HCD) with a normalized collision energy of $30 \mathrm{eV}$. MS/MS scans were performed at a resolution of 15,000 at $\mathrm{m} / \mathrm{z} 200$ with an AGC Target of $100 \%$ and a maximum injection time of $28 \mathrm{~ms}$. Dynamic exclusion was set to $30 \mathrm{~s}$ to avoid repeated sequencing of identical peptides. Each column was equilibrated with two 120 min HeLa runs before the representative run for column cross-comparison.

\section{Data analysis}

MS raw files were analyzed by MaxQuant software, version 1.6.11.0, and peptide lists were searched against the human Uniprot FASTA database. A contaminant database generated by the Andromeda search engine was configured with cysteine carbamidomethylation as a fixed modification and $\mathrm{N}$-terminal acetylation and methionine oxidation as variable modifications. We set the false discovery rate (FDR) to 0.01 for protein and peptide levels with a minimum length of 7 amino acids for peptides and the FDR was determined by searching a reverse database. Enzyme specificity was set as C-terminal to arginine and lysine as expected using trypsin and LysC as proteases. A maximum of two missed cleavages were allowed. Peptide identification was performed with an initial precursor mass deviation up to $7 \mathrm{ppm}$ and a fragment mass deviation of 20 ppm. All proteins and peptides matching to the reversed database were filtered out. 


\section{Bioinformatics analysis}

Bioinformatics analyses were performed in Python (version 3.6.4.) using Numpy (1.19.2), Pandas (1.1.4), Matplotlib (3.3.2), Seaborn (0.11.0) and Scipy (1.5.2) packages.

\section{Experimental design and statistical rationale}

The overall experimental design was focused on making different capillary columns for proteomics experiments as comparable as possible. To achieve this, statistical analysis was done from triplicate experiments for the packing time and pressure performance experiments. Experimental conditions for column cross-comparisons were chosen to eliminate outer influences, including measurements on the similar LC and MS system and equilibration procedures.

\section{Results and discussion}

\section{A high-pressure packing chamber for high density bead-slurries}

A central challenge of nano-flow chromatography in proteomics laboratories is the constant demand for new capillary columns. Due to their costs, commercial columns cannot be treated as a disposable item. However, in our hands, we frequently observe highest performance only for a short lifespan for ultra-high-performance applications. Therefore, to reach the needed quantity and cost requirements, we and many other laboratories produce their own capillary columns. However, the throughput of production is limited, especially for columns with small inner diameter and extended length such as the $50 \mathrm{~cm} 75 \mu \mathrm{m}$ inner diameter columns used in most applications in our laboratories. We produce pulled or fritted capillaries and pack them with solid phase material, typically sub $2 \mu \mathrm{m}$ C18 beads. A skilled person can pull hundreds of empty columns within a day and fritted columns are also easy to produce. However, the packing process is an inherently low-throughput and error-prone process, which makes high-performance columns prized items in mass spectrometry laboratories. Particularly achieving longer columns length is - in our experience - a precondition for ultra-high-performance.

We hypothesized that high-throughput packing of capillary columns could be achieved by highly concentrated beadslurries (21) in combination with very high-pressure packing (>1000 bar) (20). However, increased packing pressure and bead-slurry concentration can lead to column blocking, slowing down and eventually halting the packing procedure. Chloroform as a bead-solvent was reported as an approach to avoid this issue, because it can solvate higher bead concentrations. However, in combination with our bead-particles, we observed poor chromatographic performance during proteomic experiments. Instead, we combined elevated packing pressure with the FlashPack system (21), which prohibited bead aggregation at the column entrance via stirring.

To test our concept, we constructed a custom-made chamber for high-pressure packing, where the pressure derives from a conventional HPLC system (EASY-LC 1000 in our case). The device consists of a central chamber, containing the bead slurry and magnetic stirring bar, and has three openings. A large-bore access allows filling the chamber with the bead-slurry, a micro-bore fitting holds the capillary entrance into the chamber and a nano-viper connection is used as an inlet for the pressure from the HPLC system (Suppl. Fig. 1). The prototype packing chamber enabled us to fill single capillaries within minutes using the HPLC high-pressure pumps (950 bar). However, this system was not suited for high-throughput column production and the low pump volume of the HPLC system resulted in a non-continuous packing as the pump had to be refilled several times until a column was filled with beads. 


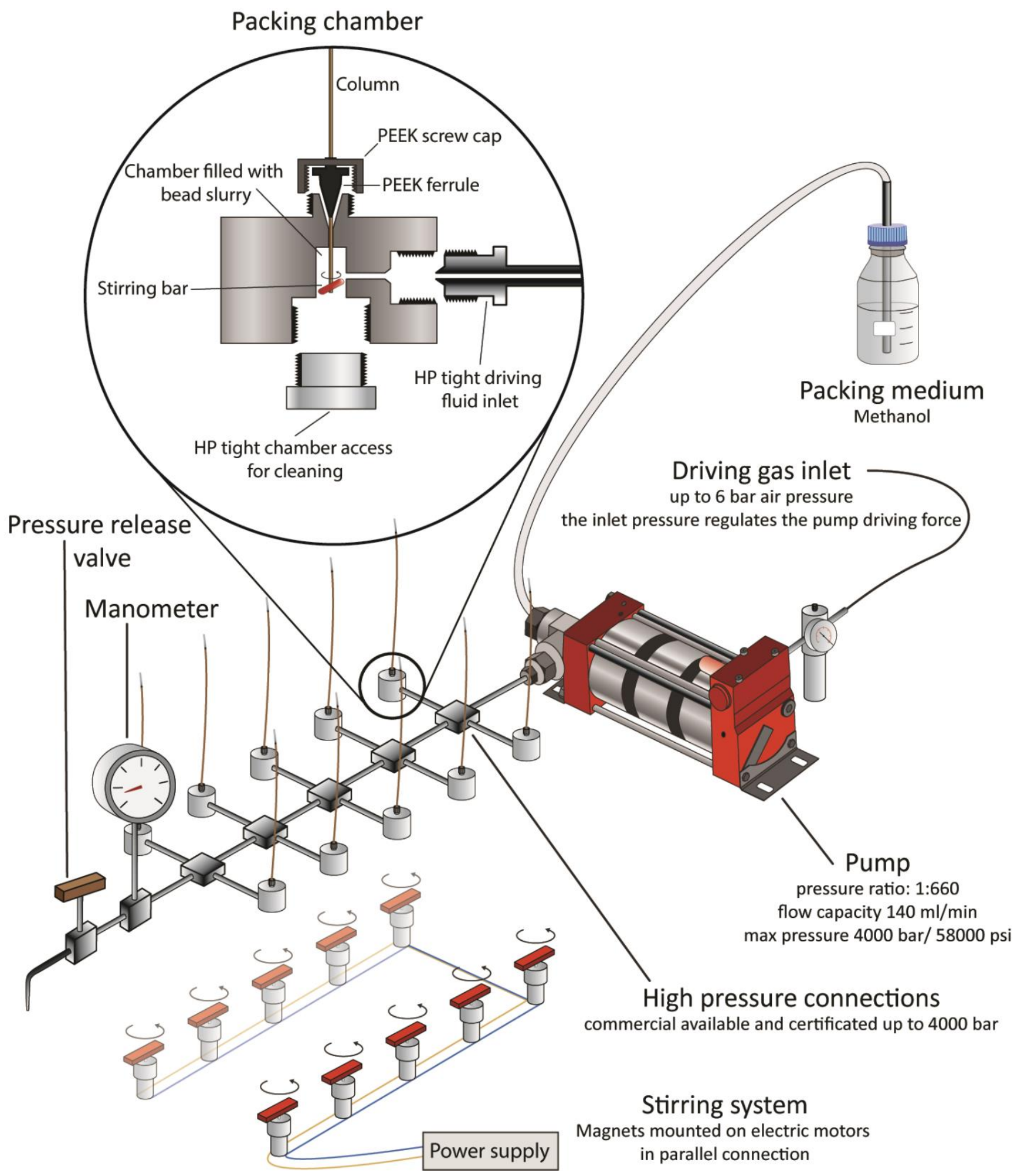

Fig. 1: High-pressure packing station. Scheme of the high-pressure packing station with detailed description of the crucial parts. The high-pressure pump is powered by a driving gas inlet and increases the pressure of a packing medium that is provided in a large volume flask by 660 -fold. The compressed packing medium is channeled to ten packing chambers, placed on top of a magnetic stirring rack. A manometer is installed to monitor the system pressure as well as a pressure release valve to facilitate time efficient system depressurization. The inset depicts a packing chamber in detail, including highpressure fittings, stirring bar and capillary column. 
Encouraged by aspects of our newly devised packing system, we set out to further streamline column production. We replaced the small volume HPLC pump with a Maximator HD-pump (Experimental Methods). This highflow continuous system converts driving gas from a standard laboratory gas supply line at a pressure ratio of 1:660 to a fluid outlet with a maximal pressure rating of 4000 bar and maximal flow capacity of $140 \mathrm{ml} / \mathrm{min}$ (Fig 1). To use the FlashPack principle we used methanol as the packing medium, which settles $\mathrm{C} 18$ beads at the chamber bottom. The high flow capacity allowed us to implement multiple pump outlets for multiplex packing of up to ten columns with our station. We redesigned the original packing chamber to fit high-pressure connections (Suppl. Fig. 2). For optimal stirring, we further created a rack system with magnets mounted on electric motors via 3D printed components to fit directly underneath the packing stations (Detailed in Experimental Methods and Suppl. Fig. 3). Moreover, we connected a high-pressure range manometer to monitor packing pressure and added a pressure relief valve for efficient and controlled depressurization of the system, a notoriously timeconsuming process. Even though the system is typically running at 1500 bar in our laboratory, the relief of pressure takes only 60 seconds, without flow-back from the running beads from the capillary. Additionally, the system is secured from capacity exceeding driving gas pressure by a control valve, which prevents the pump to be exposed to higher input than 6 bar. As with conventional packing systems, the weakest connection is the sealing of the capillary to the high-pressure chamber. We used a standard polyether-ether-ketone (PEEK) ferrule employed in HPLC applications in combination with a newly designed, reinforced PEEK screw cap (Suppl. Fig. 2D) to pin the column under very high pressure. Nevertheless, if the system pressure exceeds the durability of the material, the column is ejected. Due to the low compression capabilities of methanol, this is dangerous if one has body parts directly above the fitting when a rupture occurs and this must be prevented. Compared to gas, which can compress much more than liquid, no explosion risk should arise from our new packing station. Nevertheless, our recommendation is to use this device only within a secured area.

\section{Ultra-fast column packing}

The time required to fill a capillary column with beads depends on two variables, the bead concentration of the packing slurry and the flow rate through the capillary. Empty capillaries with pulled electrospray emitter have high flow rates in the $\mu \mathrm{l} / \mathrm{min}$ range even for conventional gas-based packing bombs with lower pressure ( $<100$ bar). However, as the bead bed grows, the flow rate through the column decreases drastically. Hence, the high-density bead slurry of FlashPack enables short packing times especially for shorter columns (21). We anticipated that combining this principle with the potentially high flow rates of our extremely high-pressure system would significantly reduce packing times.

To quantify the production throughput of our system, we consecutively packed $50 \mathrm{~cm}$ capillaries with $75 \mu \mathrm{m}$ ID at different pressures (1000-2500 bar) and measured the time required. With a freshly filled bead reservoir, packing at the lowest tested pressure took on average $4.7 \mathrm{~min}$. Increasing pressure to 2000 bar results in packing times just over a minute. Even higher pressure did not result in faster packing. Overall our system decreased the time for making a single column 10 - to 100 -fold compared to previous packing procedures $(20,21)$ (Fig. $2 A-B)$. Of note, the total production throughput is even higher due to multiplexpacking and the option to quickly exchange capillaries and bead slurries. This results in a 40-800 times faster column production (Fig. 2C). Once filled with bead-slurry and mounted on the high-pressure system, the packing 
A

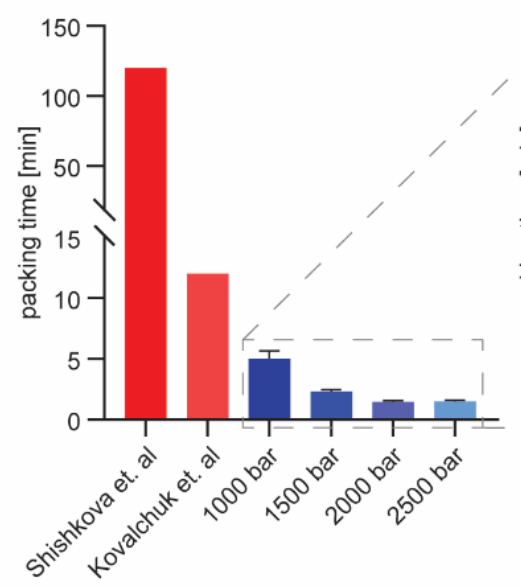

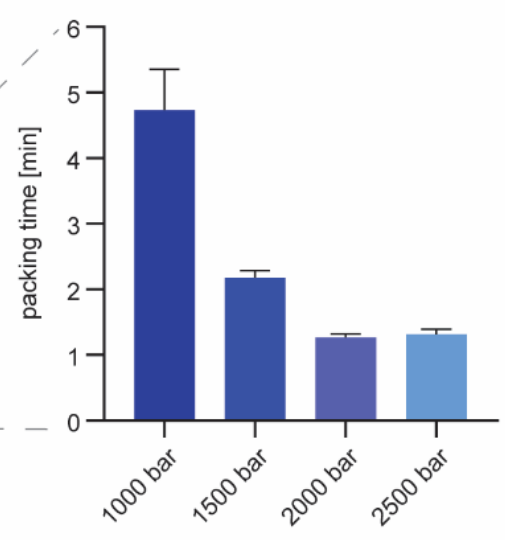

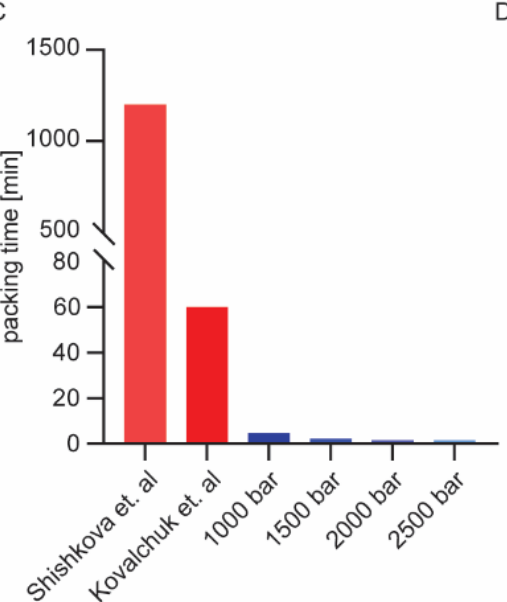

D

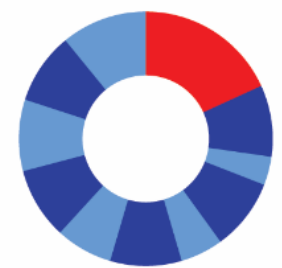

Total: 100 minutes

reservoir filling exchange of capillaries packing

Fig. 2: Comparison of packing times. A Packing times of single columns as described in previous efforts and for different packing pressures (data collected in triplicates, displayed with standard deviation) with a detailed view of the tested pressure conditions (B). C Production time for 10 columns considering multiplexing (2x multiplexing for Kovalchuk et al. and 10x for the system presented here) (20,21). D Times of a packing cycle of $10 \times 5$ columns, taking a total of 100 minutes with filling of the reservoir and changing of capillaries between the actual packing steps.

chambers can be used to pack several columns consecutively. This merely requires depressurizing the system via the pressure relief valve and exchange the filled columns with empty capillaries. Consecutive packing of several columns from the same reservoir will decrease the packing speed due to the removal of beads from the reservoir. To fully restore packing speed, the bead chamber has to be opened and refilled, which takes about $10 \mathrm{~min}$ for all ten chambers together. Typically, we refilled the reservoir after five capillary exchanges. The average turnaround cycle for producing ten columns is thus 20 minutes, allowing the production of hundreds of columns in a working day (Fig. 2D). An additional advantage of the highthroughput system is that it allows us to discard nonproperly packed columns, which occur in approximately $10 \%$ of cases.

The high-pressure system faces the same two main challenges as other packing stations, which are particle clogging within the capillary and bead aggregation at the column entrance. Particle clogging can only be avoided by clean working conditions. This means dust free storage and clean cutting of fused silica and the use of filtered fluids and dust free particles for bead slurry preparation. Bead aggregation from dense slurry can be circumvented by optimized stirring conditions according to the FlashPack principle (21).

\section{Influence of packing pressure on column performance}

To evaluate the effect of packing pressure on column performance on realistic samples, we analyzed three of our laboratory standard HeLa digests on each column. Across all packing conditions, we observed no significant variation in the number of identified peptides and protein groups (Fig. $3 A / B)$. Moreover, the median peak widths of identified peptides were comparable for all conditions (Fig. 3C). Correlation between the non-corrected retention times of peptides analyzed using columns produced at varying pressures was remarkably high (Pearson correlation coefficients > 0.996) and not significantly altered from replicates packed with similar pressure conditions (Fig. 3D). 
A

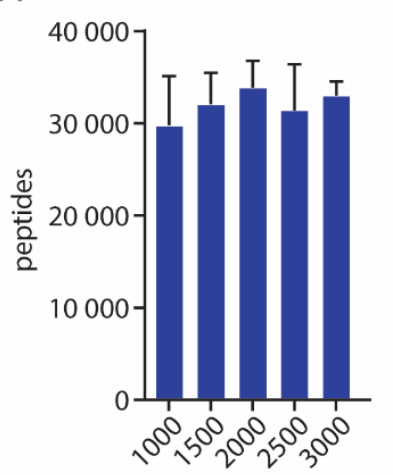

packing pressure $[\mathrm{bar}]$

D

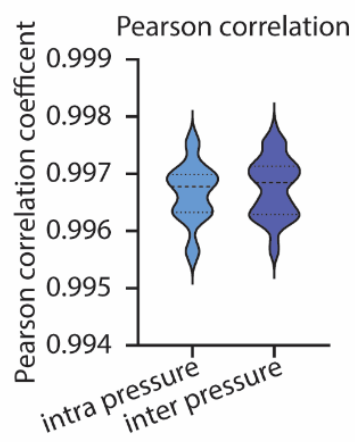

B

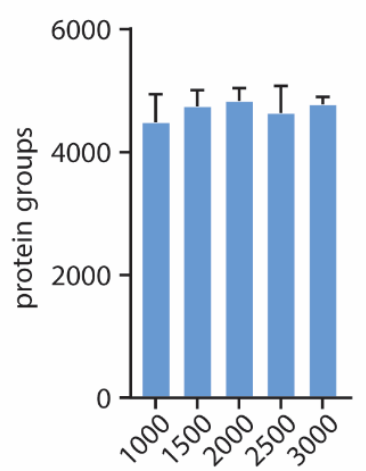

packing pressure $[\mathrm{bar}]$

$\mathrm{E}$

Tailing factor $=\frac{\text { Retention length }}{2 \times\left(\mathrm{RT}_{\text {APEX }}-\mathrm{RT}_{\text {start }}\right)}$

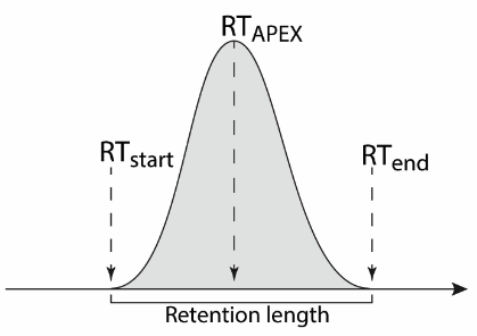

binned peptide retention length [min]
C

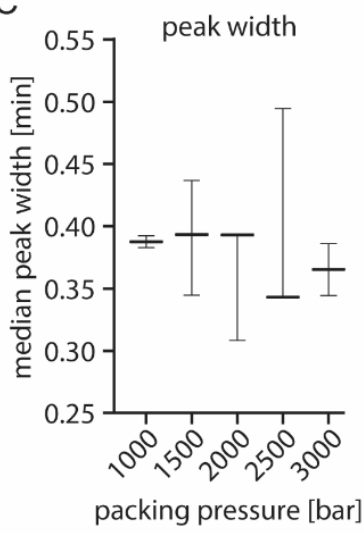

F

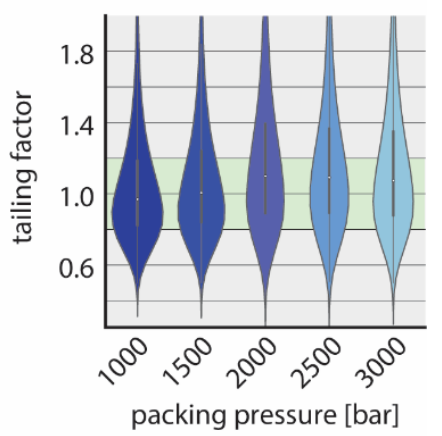

G

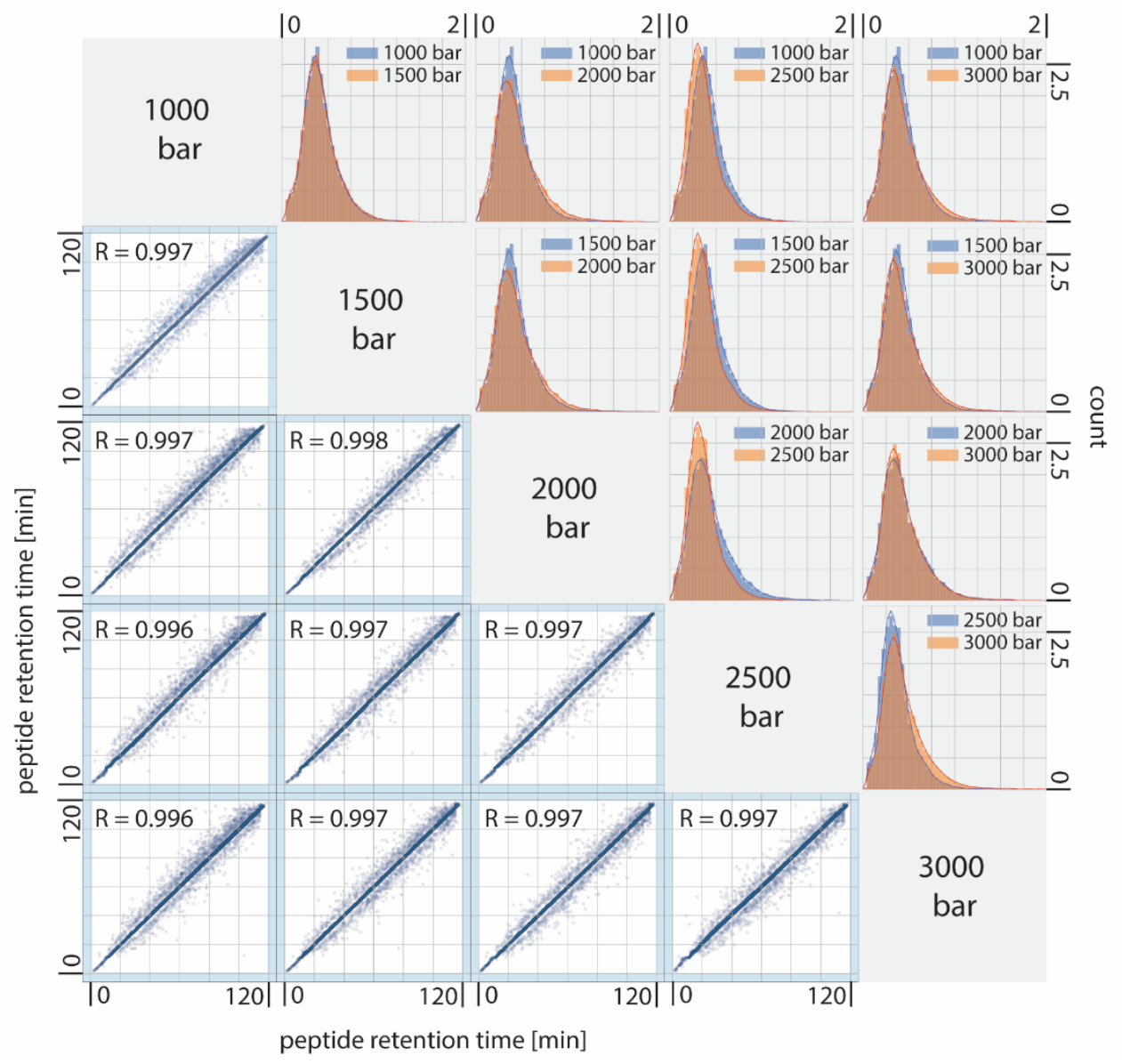


Fig. 3: Comparison of capillary columns packed at different pressures. A, Numbers of identified peptides of triplicate measurements of 500 ng HeLa digests on columns filled at the indicated packing pressures. Peptides were separated on $50 \mathrm{~cm}, 75 \mu \mathrm{m}$ ID columns packed with $1.9 \mu \mathrm{m}$ Reprosil $\mathrm{AQ}$ Beads (Dr. Maisch) with a 2-hour gradient. B, Numbers of identified protein groups of the same conditions as in (A). Error bars indicate the standard deviation from triplicate measurements. C, Median peak widths of identified peptides. D, Distribution of Pearson correlation coefficients calculated on peptide retention times between columns packed at the same pressure and columns packed at different pressures ( $p$-value of unpaired t-test for difference: 0.6 ). E, Visualization of the tailing factor calculation. F, Tailing factors for all identified peptides from runs with $75 \mu \mathrm{m}$ ID columns and different packing pressures. G, Correlation of peptide retention times across packing conditions. The density of peptides is color-coded. The histograms show the peak width distribution of four representative runs.

Another factor often used to characterize column performance is the tailing factor which can be calculated as depicted in figure $3 E(23)$. Usually, the peak width at $5 \%$ peak height is used for peak width calculation but in proteomics experiments where tens of thousands of peaks are investigated, the base-to-base peak width is typically calculated, although full width at half maximum (FWHM) is also often reported. In general, the distribution of peak shapes was wider than what would be expected from an analysis run of few analytes, but the median typically centered around the optimum of 1 . The median of the tailing factor was below 1.0 for the lower and shifts above 1.0 for higher packing pressures up to a median of 1.2 (Fig 3F). In the literature tailing factors in the range between $1-1.2$ are often described (24). The shift towards this range with the higher packing pressures could result from denser compressed bead bed. As described above the general performance was not altered for the proteomics metrics,
Column legth comparison

A

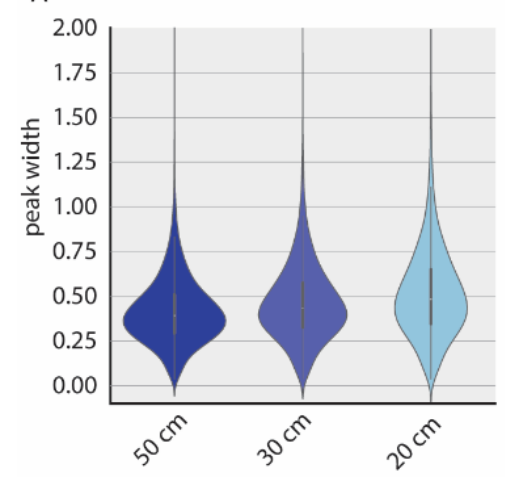

B
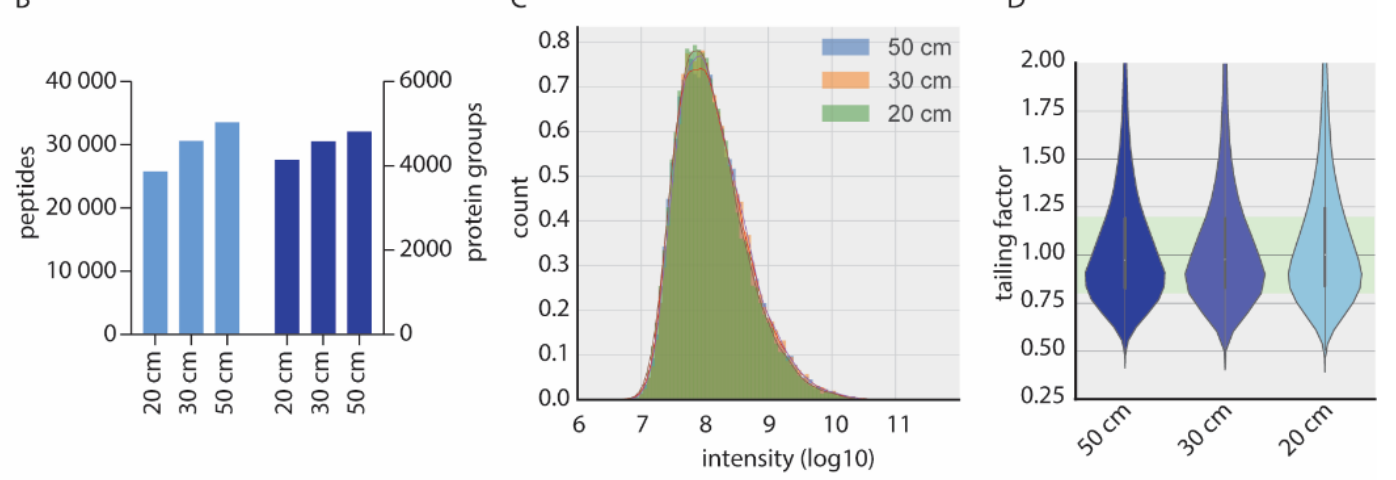

Column ID comparison$$
\text { E }
$$

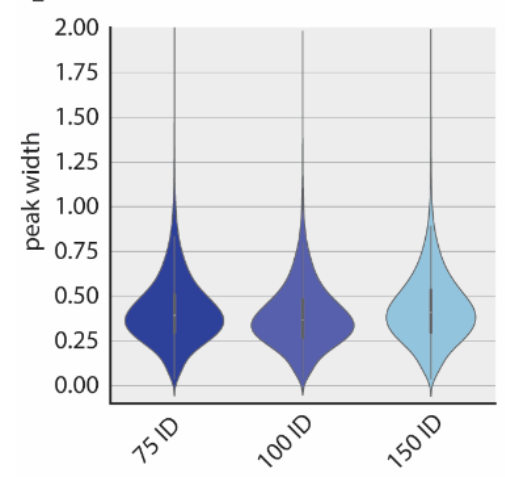

$\mathrm{F}$

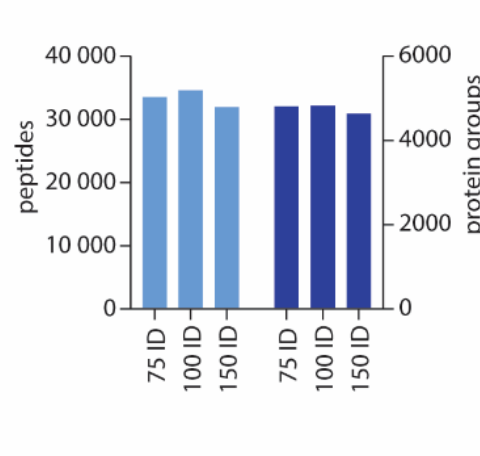

G

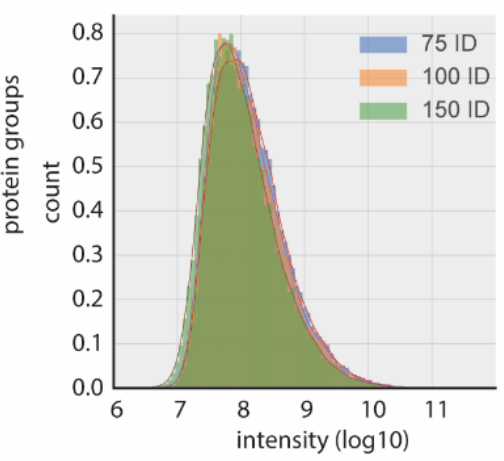

$\mathrm{H}$

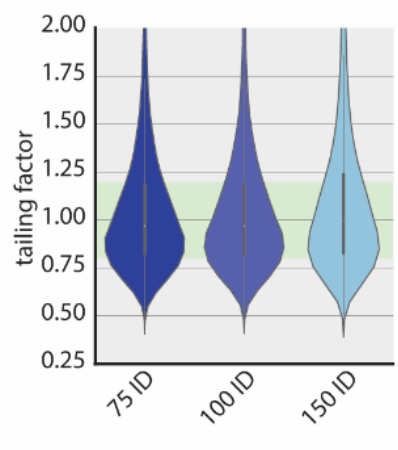

Fig. 4: Length and inner diameter comparison. All columns were packed with 1000 bar packing pressure. A, Peak width distribution from HeLa runs with different column length with the respective number of peptide and protein identifications (B), peptide intensity distribution (log10) (C) and tailing factor distribution (D). E, Peak width distribution from HeLa runs with different column IDs with the respective number of peptide and protein identifications (F), peptide intensity distribution $(\log 10)(\mathrm{G})$ and tailing factor distribution $(\mathrm{H})$. 
which leads us to the conclusion that the minor change in tailing factors with higher packing pressures is not changing the LC-MS performance. This manifests in an only slightly altered distribution of peak widths between representative experiments of columns packed at different pressures (Fig. $3 G)$. From the correlation of peptide retention times, it is visible that for all representative comparisons, the peptides elute in a narrow and reproducible time window that is not influenced by the applied packing pressure. This retention time stability is accompanied by similar separation properties of the different columns, which can be visualized directly by the retention length of analyzed molecules. Figure $3 G$ shows bulk analysis of all identified peptides with nearly overlapping retention length distributions whereas the minor differences do not constitute a significant trend towards a better performance for lower or higher packing pressures of capillary columns. Based on these results it seems that the packing pressure has no or only minimal effect on the column performance.

\section{LC-MS performance of columns with different length} and inner diameter

Length and inner diameter of capillary columns allow their adaptation to a plethora of sample materials and LC systems, specifically regarding separation power and backpressure. In MS-based proteomics, $75 \mu \mathrm{m}$ ID columns in combination with flow rates in the range of 200-400 nanoliter per minute are typical. Hence, we packed such capillary columns with different lengths $(20,30,50 \mathrm{~cm})$ with our high-pressure system and compared their performance. Packing time for the shorter columns was even faster and in the range of $30 \mathrm{sec}$. The longest columns produced the smallest peak widths and subsequently resulted in the highest numbers of identified peptides and proteins (Fig. 4A-B). Interestingly, the distribution of peptide intensities did not change significantly, and the tailing factor also remained unaffected (Fig 4C-D). Over the last years the demand for high-throughput analysis has become apparent for the analysis of clinical samples, especially blood plasma as we have described before (25). This has been addressed by a novel HPLC principle with pre-formed gradients and slightly higher flow rates (18) and by higherflow systems operating in the upper microliter per minute range $(10,26)$. As these strategies require columns with higher inner diameter to maintain acceptable pressure during analysis, we produced columns with $75 \mu \mathrm{m}, 100 \mu \mathrm{m}$ and $150 \mu \mathrm{m}$ ID and tested their performance.

When comparing column inner diameters, the experimental setup has to be adapted to the conditions. To enable direct comparison of capillaries with different ID, we scaled the flow rates to reach the same linear velocities and the amount of input material to the column volume (Experimental Procedures). For the $100 \mu \mathrm{m}$ ID columns this results in a flow rate of $535 \mathrm{nl} / \mathrm{min}$ and $888 \mathrm{ng}$ of peptides for loading, whereas for the $150 \mu \mathrm{m} \mathrm{ID} \mathrm{column} 1200 \mathrm{nl} / \mathrm{min}$ and $2 \mu \mathrm{g}$ of peptide material was loaded to be comparable to the $300 \mathrm{nl} / \mathrm{min}$ and $500 \mathrm{ng}$ employed for the $75 \mu \mathrm{m}$ ID columns. This requirement of higher sample amount already limits the applicability of larger column diameters for samples with limited accessibility. The $1400 \mu \mathrm{l}$ pump volume of the Easy-LC 1200 used for the experiment were sufficient to run a 2-hour gradient with the $150 \mu \mathrm{m}$ ID column, but longer gradients or higher flow rates would exceed the capabilities of the LC-system and require higher flow rates. The larger column IDs led to slightly broader peak widths, but peptide and protein identifications were not affected. Due to the correction of the sample input amount, we have not seen a difference in peptide intensity distributions, and the peak tailing has not been affected by the column ID (Fig 4E-H). 


\section{Conclusion}

Here, we aimed to increase the throughput and to streamline the production of capillary columns for MSbased proteomics. We provide a detailed list for commercial parts and blueprints describing the construction of our highpressure packing station. The setup can be built at relatively low costs $(<\$ 10,000)$, compared to the cumulative expenses for high performing commercial columns. We designed this new station to fill multiple columns simultaneously within a few minutes, which accelerates the packing process of capillary columns more than 100 -fold compared to traditional gas pressure driven stations. In this way, we hope our system helps researchers streamlining the often work-intensive and fragile column production process. In addition, the extreme high pressures enable the packing of long, high-performing columns $(>50 \mathrm{~cm})$. The ability to produce high-performing columns at highthroughput opens up the possibility of using capillary columns always at the peak of their performance, replacing them as soon as peak-broadening or decreased ionization is observed. Reassuring in terms of the robustness of the packing process itself and the stability achieved at exceedingly high pressures, we have not observed variation in the performance characteristics over a wide range of packing pressure from 1000 to 3000 bar. We hope the technology described here will enable laboratories of any scale to mass-produce high performance long capillary columns.

\section{Literature}

1. Aebersold R, Mann M. Mass spectrometry-based proteomics [Internet]. Vol. 422, Nature. Nature Publishing Group; 2003 [cited 2020 Dec 15]. p. 198-207. Available from: www.nature.com/nature

2. Aebersold R, Mann M. Mass-spectrometric exploration of proteome structure and function. Nature [Internet]. 2016 Sep 15 [cited 2019 Apr 21];537(7620):347-55. Available from: http://www.nature.com/articles/nature19949

3. Michalski A, Cox J, Mann M. More than 100,000 detectable peptide species elute in single shotgun proteomics runs but the majority is inaccessible to datadependent LC-MS/MS. J Proteome Res [Internet]. 2011 Apr 1 [cited 2021 Feb 20];10(4):1785-93. Available from: https://pubs.acs.org/sharingguidelines

4. Shishkova E, Hebert AS, Coon JJ. Now, More Than Ever, Proteomics Needs Better Chromatography. Vol. 3, Cell Systems. Cell Press; 2016. p. 321-4.

5. Horváth C, Melander W, Molnár I. Solvophobic interactions in liquid chromatography with nonpolar stationary phases. J Chromatogr A. 1976 Sep 29;125(1):129-56.

6. Fenn JB, Mann M, Meng CK, Wong SF, Whitehouse CM. Electrospray ionization for mass spectrometry of large biomolecules. Science. 1989.

7. Kennedy RT, Jorgenson JW. Preparation and Evaluation of Packed Capillary Liquid Chromatography Columns with Inner Diameters from 20 to $50 \mu \mathrm{m}$. Anal Chem [Internet]. 1989 [cited 2021 Feb 20];61(10):1128-35. Available from: https://pubs.acs.org/sharingguidelines

8. Emmett MR, Caprioli RM. Micro-Electrospray Mass Spectrometry: Ultra-High-Sensitivity Analysis of Peptides and Proteins [Internet]. 1994 [cited 2021 Feb 20]. Available from: https://pubs.acs.org/sharingguidelines

9. Ishihama Y, Rappsilber J, Andersen JS, Mann M. Microcolumns with self-assembled particle frits for proteomics. J Chromatogr A [Internet]. 2002 Dec 6 [cited 2020 Oct 29];979(1-2):233-9. Available from: https://linkinghub.elsevier.com/retrieve/pii/S0021967302 014024

10. Bian $Y$, Zheng R, Bayer FP, Wong C, Chang YC, Meng $\mathrm{C}$, et al. Robust, reproducible and quantitative analysis of thousands of proteomes by micro-flow LC-MS/MS. Nat Commun. 2020 Dec 1;11(1).

11. Bian Y, Bayer FP, Chang Y-C, Meng C, Hoefer S, Deng $\mathrm{N}$, et al. Robust Microflow LC-MS/MS for Proteome Analysis: 38000 Runs and Counting. Anal Chem [Internet]. 2021 Feb 17 [cited 2021 Feb 
22];acs.analchem.1c00257. Available from: https://pubs.acs.org/doi/10.1021/acs.analchem.1c00257

12.

Bernhardt O, Selevsek N, Gillet L, Rinner O, Picotti P, Aebersold R, et al. $<\mathrm{p}>$ Spectronaut: a fast and efficient algorithm for MRM-like processing of data independent acquisition (SWATH-MS) data $</ p>$. F1000Research. 2014 Aug 14;5.

13. Cox J, Mann M. MaxQuant enables high peptide identification rates, individualized p.p.b.-range mass accuracies and proteome-wide protein quantification. Nat Biotechnol [Internet]. 2008 Dec 30 [cited 2019 Apr 21];26(12):1367-72. Available from: http://www.nature.com/articles/nbt.1511

14. Hu Q, Noll RJ, Li H, Makarov A, Hardman M, Cooks RG. The Orbitrap: A new mass spectrometer [Internet]. Vol. 40, Journal of Mass Spectrometry. John Wiley \& Sons, Ltd; 2005 [cited 2021 Jan 20]. p. 430-43. Available from: https://onlinelibrary.wiley.com/doi/full/10.1002/jms.856

15. Kelstrup CD, Bekker-Jensen DB, Arrey TN, Hogrebe A, Harder A, Olsen J V. Performance Evaluation of the $Q$ Exactive HF-X for Shotgun Proteomics. J Proteome Res [Internet]. 2018 Jan 5 [cited 2020 Dec 15];17(1):727-38. Available from: https://pubs.acs.org/sharingguidelines

16. Meier F, Beck S, GrassI N, Lubeck M, Park MA, Raether $O$, et al. Parallel accumulation-serial fragmentation (PASEF): Multiplying sequencing speed and sensitivity by synchronized scans in a trapped ion mobility device. J Proteome Res [Internet]. 2015 [cited 2021 Jan 20];14(12):5378-87. Available from: https://pubmed.ncbi.nlm.nih.gov/26538118/

17. Meier F, Brunner AD, Frank M, Ha A, Bludau I, Voytik E, et al. diaPASEF: parallel accumulation-serial fragmentation combined with data-independent acquisition. Nat Methods [Internet]. 2020 Dec 1 [cited 2021 Jan 20];17(12):1229-36. Available from: https://doi.org/10.1038/s41592-020-00998-0

18. Bache N, Geyer PE, Bekker-Jensen DB, Hoerning O, Falkenby $L$, Treit $P$ V., et al. A novel LC system embeds analytes in pre-formed gradients for rapid, ultra-robust proteomics. Mol Cell Proteomics. 2018 Nov 1;17(11):2284-96.

19. Richards AL, Merrill AE, Coon JJ. Proteome sequencing goes deep. Vol. 24, Current Opinion in Chemical Biology. Elsevier Ltd; 2015. p. 11-7.

20. Shishkova E, Hebert AS, Westphall MS, Coon JJ. UltraHigh Pressure $(>30,000$ psi) Packing of Capillary Columns Enhancing Depth of Shotgun Proteomic Analyses. Anal Chem [Internet]. 2018 Oct 2 [cited 2020 Oct 29];90(19):11503-8. Available from: https://pubs.acs.org/sharingguidelines
21. Kovalchuk SI, Jensen ON, Rogowska-Wrzesinska A. FlashPack: Fast and simple preparation of ultrahighperformance capillary columns for LC-MS. Mol Cell Proteomics [Internet]. 2019 Feb 1 [cited 2020 Dec 15];18(2):383-90. Available from: https://www.mcponline.org

22. Bruns S, Franklin EG, Grinias JP, Godinho JM, Jorgenson $J W$, Tallarek U. Slurry concentration effects on the bed morphology and separation efficiency of capillaries packed with sub- $2 \mu \mathrm{m}$ particles. J Chromatogr A [Internet]. 2013 Nov 29 [cited 2020 Oct 30];1318:189-97. Available from:

https://linkinghub.elsevier.com/retrieve/pii/S0021967313 016178

23. Analytical Separation Science. Analytical Separation Science. Wiley-VCH Verlag GmbH \& Co. KGaA; 2015.

24. Birdsall RE, Kellett J, Yu YQ, Chen W. Application of mobile phase additives to reduce metal-ion mediated adsorption of non-phosphorylated peptides in RPLC/MSbased assays. J Chromatogr B Anal Technol Biomed Life Sci. 2019 Sep 15;1126-1127:121773.

25. Geyer PE, Holdt LM, Teupser D, Mann M. Revisiting biomarker discovery by plasma proteomics. Mol Syst Biol [Internet]. 2017 Sep [cited 2020 Dec 15];13(9):942. Available from: https://pubmed.ncbi.nlm.nih.gov/28951502/

26. Messner C, Demichev V, Bloomfield N, Ivosev G, Wasim $F$, Zelezniak $A$, et al. ScanningSWATH enables ultra-fast proteomics using high-flow chromatography and minutescale gradients [Internet]. bioRxiv. bioRxiv; 2019 [cited 2020 Dec 15]. p. 656793. Available from: https://doi.org/10.1101/656793

\section{Acknowledgments}

We thank all members of the Proteomics and Signal Transduction Group at the Max Planck Institute of Biochemistry and the Clinical Proteomics Group of the NNF Center for Protein Research for help and discussions and in particular Igor Paron, Christian Deiml for technical assistance, Mario Oroshi for help with the online resource. We thank the mechanical workshop and the educational workshop especially Martin Wied, Andreas Kucher and Harry Spangenberg of the Max Planck Institute of Biochemistry for the fabrication and iterative optimization of all self-constructed parts. 


\section{Funding}

The work carried out in this project was partially supported

by the Max Planck Society for the Advancement of Science.

\section{Author contributions}

J.B.M-R. designed and assembled the packing station parts and carried out the bioinformatics analyses. J.B.M-R., L.S., F.H., P.G., M.M. and P.V.T. designed the experiments, performed and interpreted the MS-based proteomic analyses, generated text and figures and wrote the manuscript. M.M. supervised and guided the project, designed the experiments, interpreted MS-based proteomics data.

\section{Competing interests}

The authors declare no competing interests. 
bioRxiv preprint doi: https://doi.org/10.1101/2021.02.26.433033; this version posted March 30, 2021. The copyright holder for this preprint (which was not certified by peer review) is the author/funder. All rights reserved. No reuse allowed without permission.

\section{Multiplexed high-pressure column packing}

\section{Supplementary Figures}
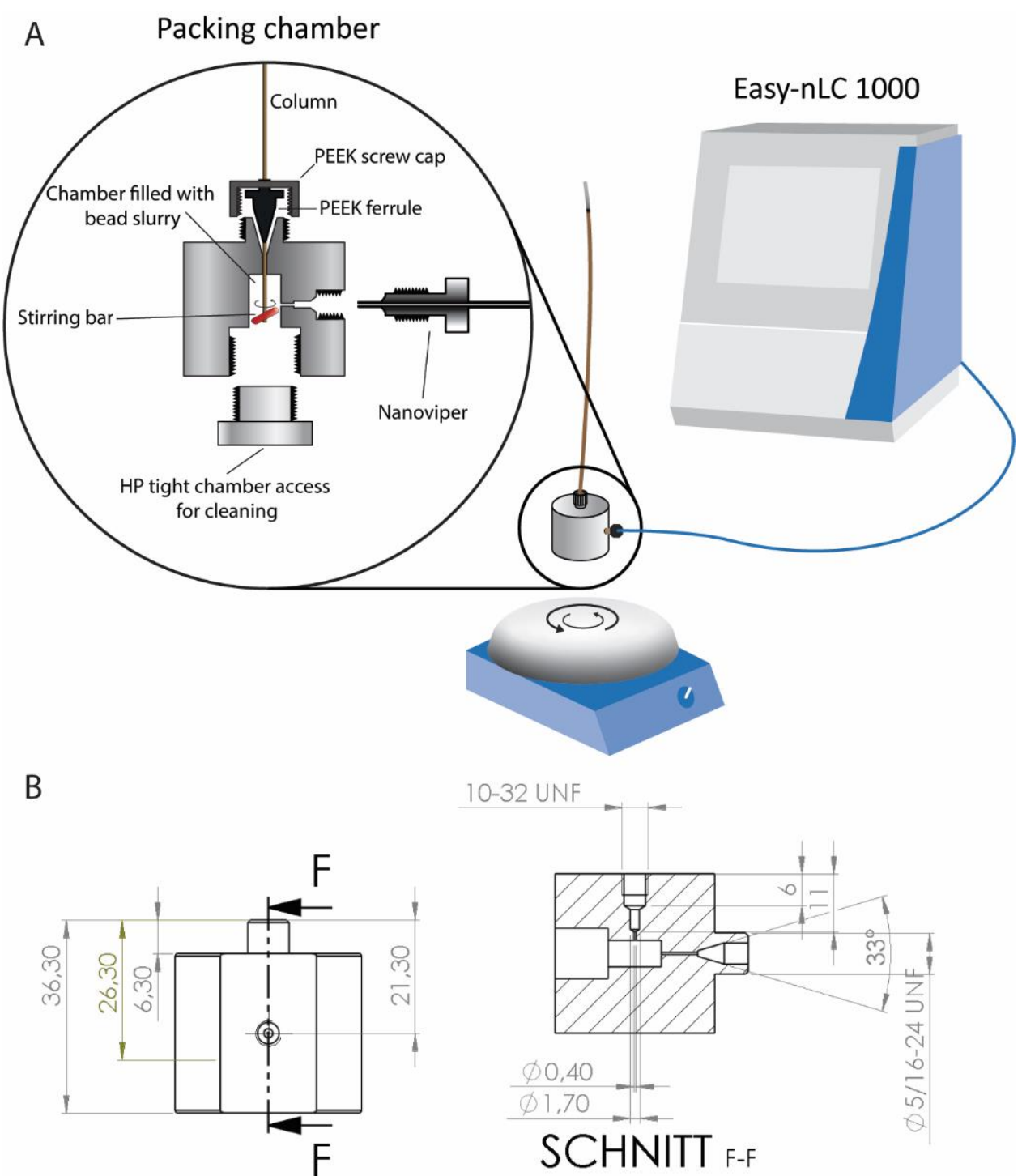

\section{0-32 UNF}
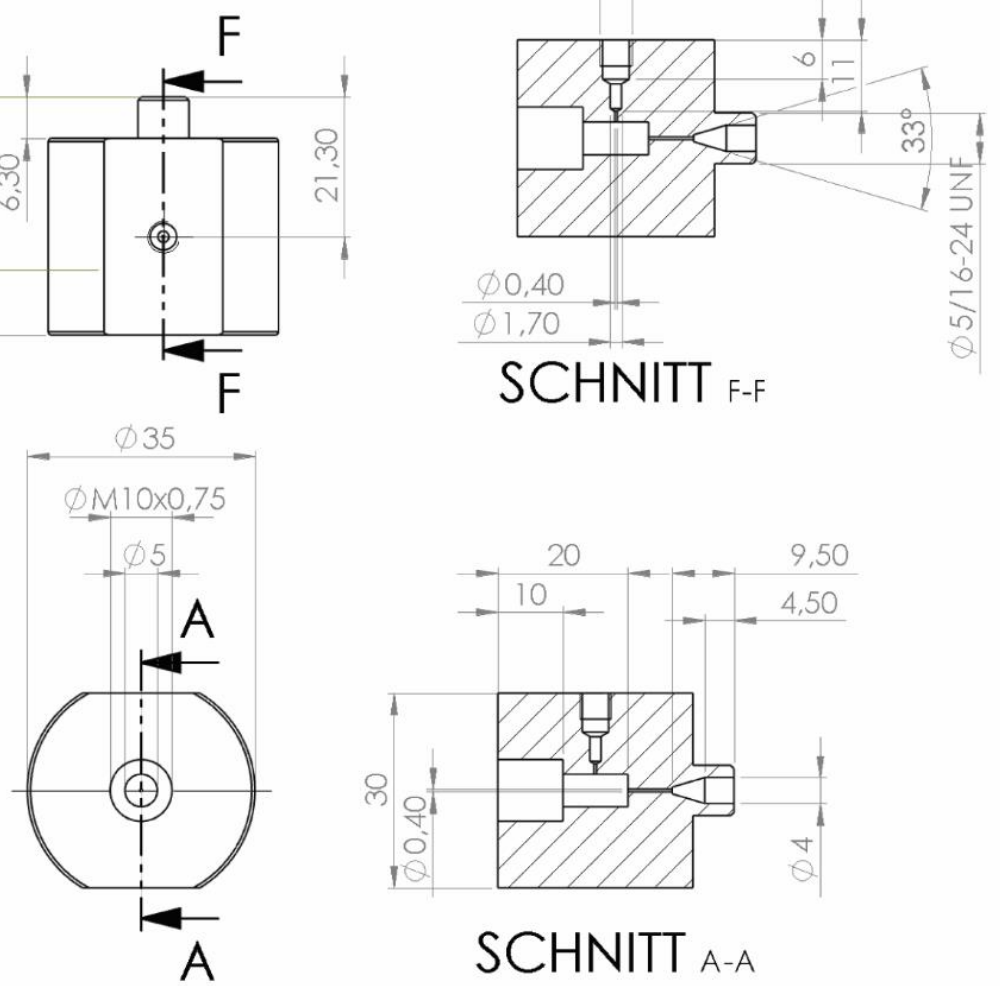

\section{SCHNITT A-A}

Suppl. Figure 1. Prototype one of the high-pressure packing chamber. A, An early prototype of the packing chamber for use with a LC system with nano-viper connection output. The station was used on top of a magnetic stirrer and supplied with driving fluid and pressure from an Easy-nLC 1000. B, Technical drawing of the standalone prototype. 
bioRxiv preprint doi: https://doi.org/10.1101/2021.02.26.433033; this version posted March 30, 2021. The copyright holder for this preprint (which was not certified by peer review) is the author/funder. All rights reserved. No reuse allowed without permission.

\section{Multiplexed high-pressure column packing}

A
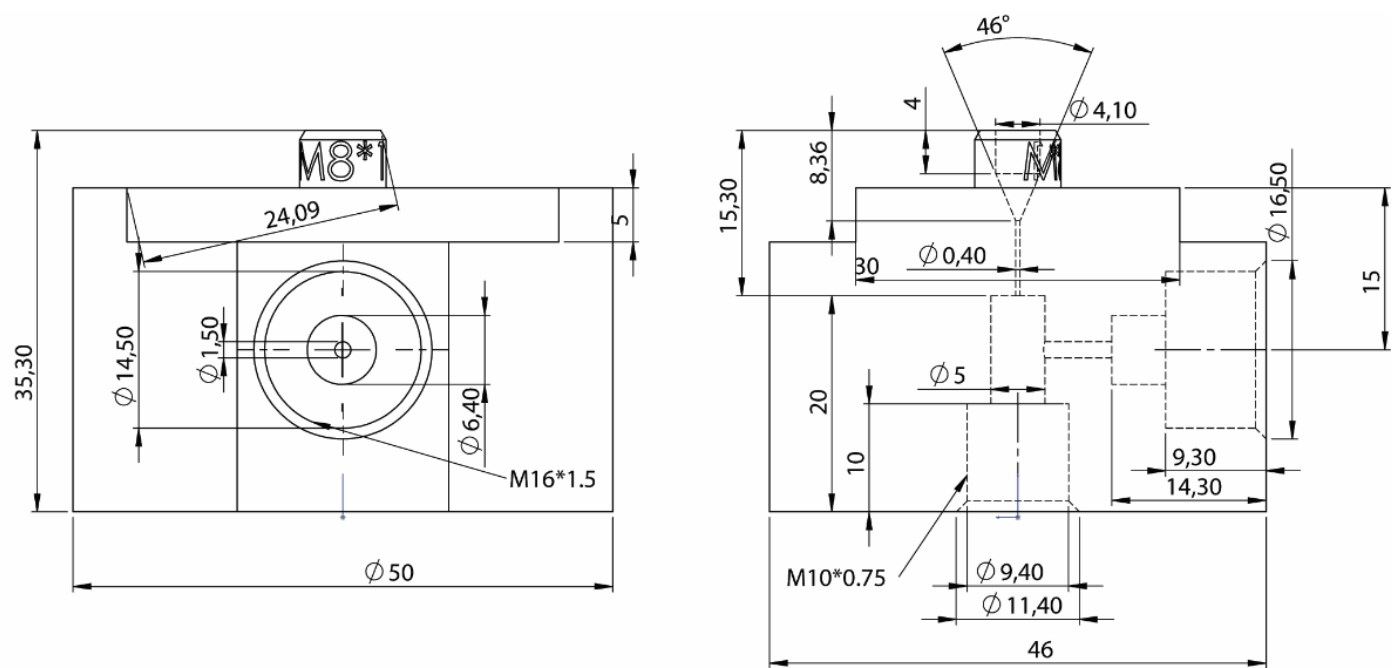

B

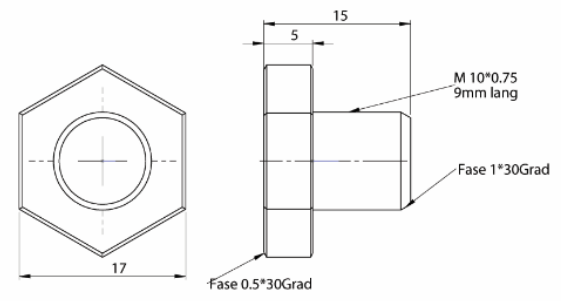

C
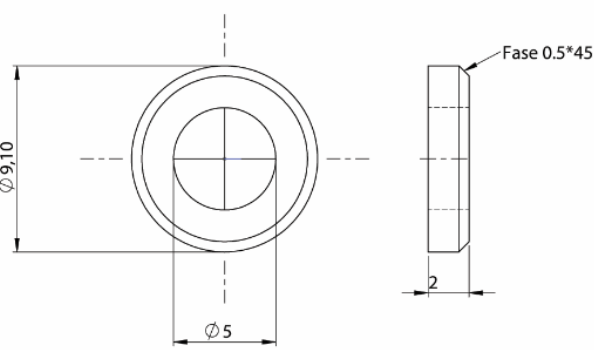

D

$\mathrm{E}$
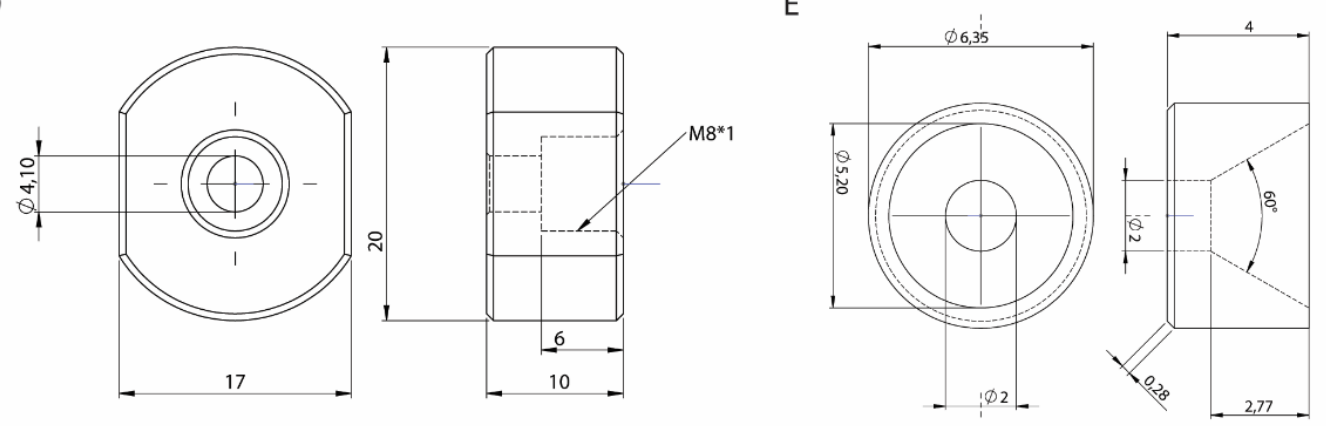

$\mathrm{F}$

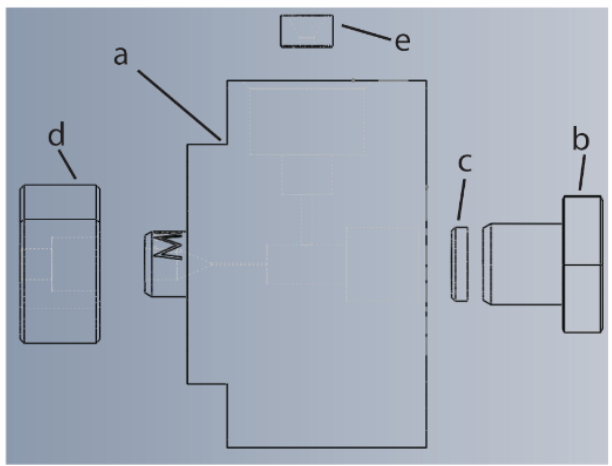

Suppl. Figure 2. Technical drawings of the packing chamber. A, Packing chamber made from stainless steel. B, Closing screw for packing chamber and prototype one (Suppl. Fig.1) made from stainless steel. C, PEEK seal ring for the closing screw. D, GFPEEK connection screw cap to press the PEEK ferrule into the coned fitting. E, PEEK seal ring for the connection to the HP supply system. F, Assembly of the parts A-E. 
bioRxiv preprint doi: https://doi.org/10.1101/2021.02.26.433033; this version posted March 30, 2021. The copyright holder for this preprint (which was not certified by peer review) is the author/funder. All rights reserved. No reuse allowed without permission.

\section{Multiplexed high-pressure column packing}

A
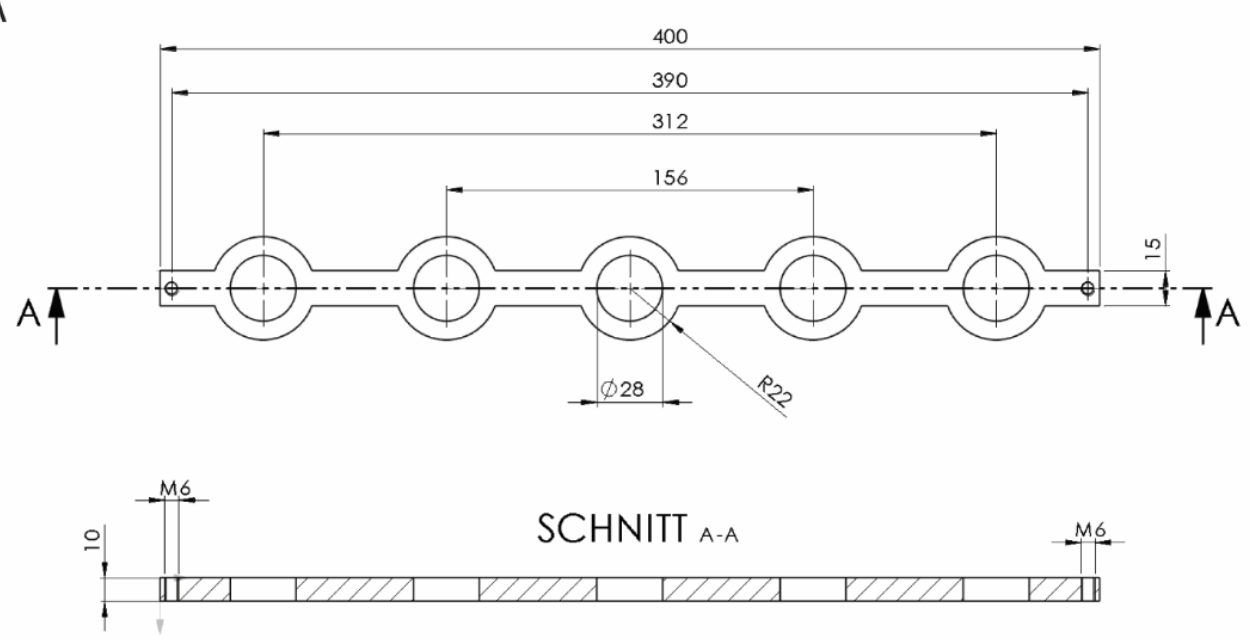

B
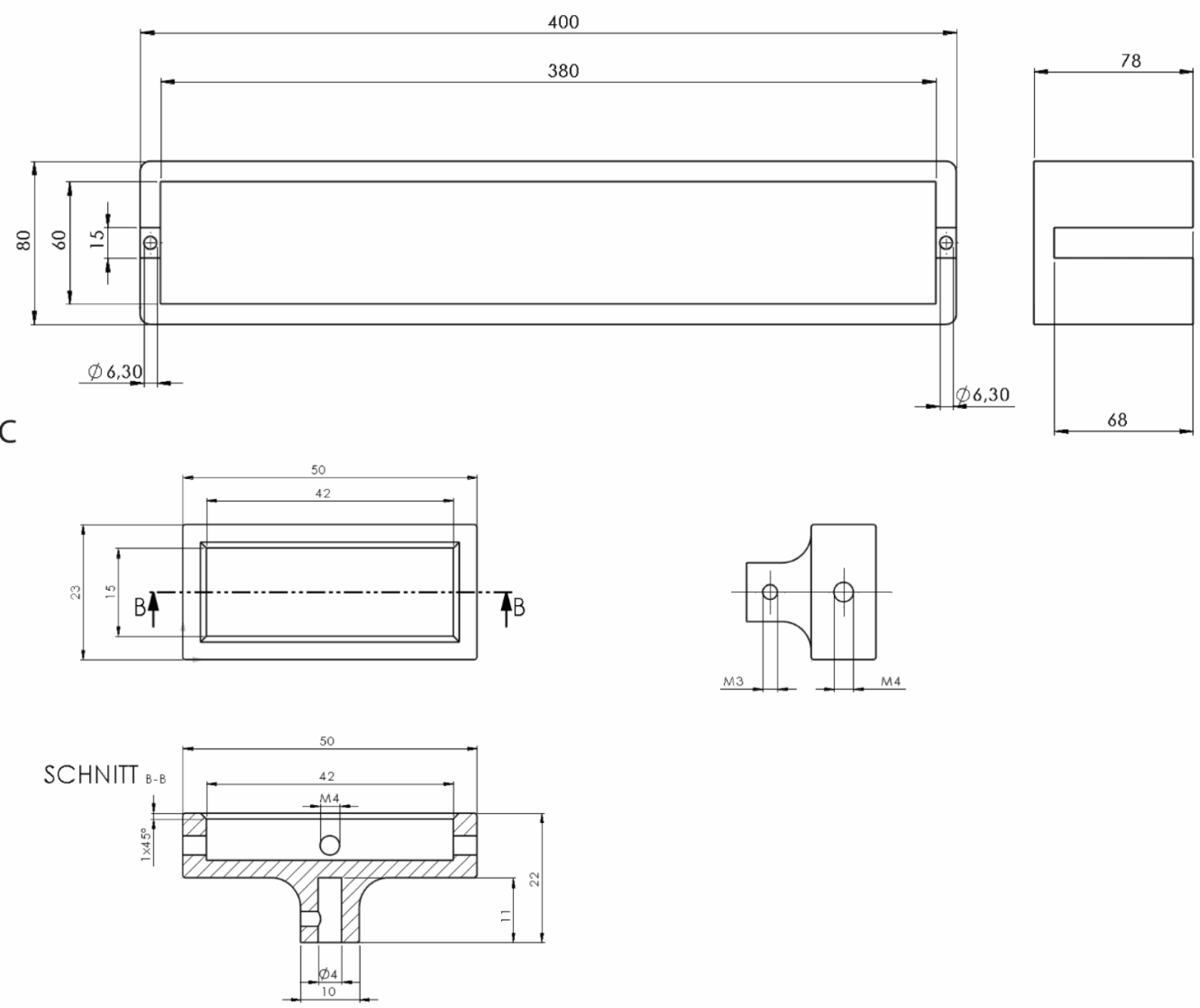

Suppl. Figure 3. Technical drawings of the stirring system. A) Frame for the electric motors. B) Hight adjustable stand for the magnetic holder frame in A. C) Magnet holder to be placed on the magnetic motors 3D-printed from carbon. 